NÚCLEO DE PESQUISA

EM GESTÃO DE SISTEMAS

DAPRODUÇÃO - NP GSP
Brazilian Journal of Production Engineering BJPE - Revista Brasileira de Engenharia de Produção

Disponivel em: http://periodicos.ufes.br/BJPE/index

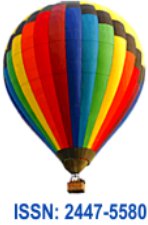

ISSN: $2447-558$
Campus São Mateus UNIVESIDADE FEDERAL DO ESPIRITO SANTO

ARTIGO ORIGINAL

OPEN ACCESS

\title{
INDÚSTRIA 4.0 APLICADA À GESTÃO DA CADEIA DE SUPRIMENTOS: UMA REVISÃO DA LITERATURA
}

INDUSTRY 4.0 APPLIED TO SUPPLY CHAIN MANAGEMENT: A LITERATURE REVIEW

\section{$\underline{\text { Gabriel Marques de Souza }^{1}}{ }^{\text {, Victor Hugo Souza de Abreu }}{ }^{2}, \mathbf{\text { Marcio de Almeida }}$ $\underline{\text { D'Agosto }}^{3}$}

123 Programa de Engenharia de Transportes do Instituto Alberto Luiz Coimbra de Pós-Graduação e Pesquisa de Engenharia, Universidade Federal do Rio de Janeiro, Av. Horácio Macedo, 2030, 101, Cidade Universitária, Rio de Janeiro, RJ, Brasil.

1* gabriel.souza@pet.coppe.ufrj.br ${ }^{2}$ victor@pet.coppe.ufrj.br ${ }^{3}$ dagosto@pet.coppe.ufrj.br

\section{ARTIGO INFO.}

\section{Recebido em: 01.04.2021}

Aprovado em: 27.04.2021

Disponibilizado em: 18.06.2021

\section{Palavras-Chave:}

Logística 4.0; Indústria 4.0; Revisão Bibliométrica.

\section{KEYWORDS:}

Logistics 4.0; Industry 4.0; Bibliometric Review.

*Autor Correspondente: Souza, G. M.

\section{RESUMO}

O desenvolvimento da Indústria 4.0 tem tornado cada vez mais populares as novas tecnologias para aperfeiçoamento das atividades relacionadas à logística e à gestão da cadeia de suprimentos, com a finalidade de aumentar a eficiência dos processos. Portanto, o presente trabalho tem como objetivo identificar as condições atuais das pesquisas sobre o tema por meio de uma revisão bibliométrica e sistemática de artigos publicados na base Web of Science, dando enfoque especial aos estudos brasileiros, que ainda estão em crescimento. Os resultados mostram a crescente relevância do tema e o interesse por parte de grandes periódicos. Em relação às publicações nacionais, percebe-se o interesse pela internet das coisas e pelos sistemas cyber-físicos. Os artigos brasileiros analisados têm como foco questões econômicas e operacionais, mas também se preocupam com questões ambientais.

\begin{abstract}
The development of industry 4.0 is popularizing the new technologies to improve logistics and supply chain management activities to increase process efficiency. Therefore, the present work aims to identify the current research conditions on the subject through a bibliometric and systematic review of papers published on the base Web of Science, giving a special focus to Brazilian studies, that is still in growth. The results show the growing relevance of the theme and the interest on the part of major journals. In relation to the national publications, there is interest in the internet of things and in the cyber-physical systems. The Brazilian analyzed papers focus on economic and operational issues, but there is also a concern about environmental issues.
\end{abstract}




\section{INTRODUÇÃO}

Observando-se os avanços tecnológicos decorrentes das primeiras três revoluções industriais, como a introdução de máquinas a vapor na primeira revolução, o advento da linha de produção na segunda e a automação de processos na terceira (Barreto et al., 2017), pode-se afirmar que as mudanças são irreversíveis, cada vez mais profundas e que as organizações que não se adaptarem se tornarão obsoletas.

Atualmente observa-se advento dos computadores autônomos conectados à internet, que possibilitam a convergência do mundo físico com o virtual, na forma de sistemas cyberfísicos, que se constitui por elementos computacionais colaborativos com o intuito de controlar entidades físicas. Assim, é possível que recursos, informações, objetos e pessoas sejam interconectados, resultando na internet das coisas (IoT) (Kagermann et al., 2013). Para diversos autores, tais como Lasi et al. (2014), Erol et al. (2016) e Sung (2018), esse pode ser considerado o quarto estágio da industrialização, sendo chamado de Indústria 4.0.

Os processos tecnológicos da Indústria 4.0 possibilitam a conexão remota de informações, objetos e pessoas devido à convergência do mundo físico e virtual no sistema cyber-físico. Além disso, também transformam as fábricas em ambientes inteligentes (Thoben et al., 2016). Neste sentido, as novas tecnologias, inseridas nesse contexto, tais como a manufatura aditiva; sistemas cyber-físicos; internet das coisas e manufatura em nuvem (Kang et al., 2016), são capazes de garantir a interoperabilidade e integração dos sistemas de produção baseando-se principalmente nas tecnologias de informação e comunicação.

Dessa forma, segundo Arnold et al. (2017) e Bauer et al. (2015), são ferramentas eficientes no desenvolvimento de soluções para os desafios que as companhias devem enfrentar para se manterem competitivas em termos de globalização e intensificação de competitividade, volatilidade de demandas do mercado, redução no ciclo de vida dos produtos e aumento da complexidade dos processos e produtos.

A introdução da Indústria 4.0 tem tido resultados de sucesso, gerando vários benefícios aos negócios, incluindo otimização operacional e das cadeias de valor (Strange \& Zucchella, 2017). O setor de logística e transportes também é passível de melhorias, baseadas principalmente numa rede de ligações entre organizações, em que os dados são integrados e os processos são organizados ao longo de uma cadeia de suprimentos, em um espaço virtual (Szozda, 2017).

A gestão da cadeia de suprimentos é uma das áreas mais importantes no que diz respeito à competitividade das organizações (Prajogo \& Sohal, 2013). Além disso, os custos logísticos representam uma grande parcela dos custos das empresas (Engblom et al., 2012). Sendo assim, a utilização de novas tecnologias nas atividades relacionadas à logística e à gestão da cadeia de suprimentos, com a finalidade de aumentar a eficiência dos processos, se mostra pertinente no contexto atual e um caminho a ser explorado no cenário brasileiro.

Assim, surge a seguinte questão: qual é o estado atual das pesquisas relacionadas à aplicação da Indústria 4.0 na gestão da cadeia de suprimentos e nas operações logísticas? A pesquisa busca também analisar as publicações brasileiras, ainda em crescimento, e avaliar os objetivos 
e abordagens dos autores nacionais em relação ao tema em questão, com o intuito de ampliar a temática no país. Portanto, este trabalho tem como objetivo apresentar um apanhado geral de artigos científicos aplicáveis ao tema, por meio de análises bibliométricas e sistemáticas, que utilizam criteriosos filtros de inclusão e qualificação de estudos.

Como objetivos específicos têm-se: (I) Apresentar a evolução das publicações e citações ao longo dos anos; (II) Identificar os principais periódicos e países que mais publicam estudos sobre o assunto; e (III) Realizar uma análise das publicações brasileiras identificadas na base de dados.

Cabe destacar que a relevância deste estudo está na ampliação do estado da arte sobre um assunto ainda novo na literatura, principalmente no contexto brasileiro, que ainda carece de pesquisas relacionadas. Inclusive durante a revisão da literatura não foi identificado nenhum estudo que trazia uma análise sobre as principais considerações expostas nas pesquisas brasileiras sobre a temática.

Visando atingir os objetivos propostos, este estudo está estruturado em 5 seções. A Seção 1 tem o objetivo de contextualizar o problema e apresentar o objetivo e a estruturação do trabalho. A Seção 2 traz uma breve revisão sobre a cadeia de suprimentos da Indústria 4.0. Na Seção 3 está descrito o procedimento metodológico utilizado nas pesquisas bibliográficas. A Seção 4 apresenta e discute os resultados. Por fim, a Seção 5 apresenta as conclusões e considerações finais, além de sugerir pesquisas futuras.

\section{IMPACTOS DA INDÚSTRIA 4.0 NA GESTÃO DA CADEIA DE SUPRIMENTOS}

Nas últimas décadas, a personalização e customização de serviços tem sido uma importante estratégia produtiva das grandes empresas. Como consequência, a complexidade dos processos envolvidos, tem aumentado e as estratégias convencionais de planejamento e gestão de suprimentos já não são o bastante (Premm \& Kirn, 2015).

A gestão da cadeia de suprimentos compreende o fluxo de materiais, de negócios, de capital e de informações (Pedroso e Nakano, 2009). De acordo com Melnyk et al. (2009), a gestão da cadeia de suprimentos tem deixado de ser uma atividade de natureza tática para, cada vez mais, se tornar uma atividade estratégica nas organizações. Nesse contexto, a logística e a gestão da cadeia de suprimentos tem ganhado mais importância, e novas tecnologias têm sido utilizadas com a finalidade de atender continuamente às demandas do mercado em termos de redução de lead time, aumento da variedade de produtos, etc. (Stock \& Seliger, 2016).

De acordo com Fernandez e Jiménez (2017), a integração na cadeia de suprimentos é fundamental e todo o processo deve ser visto com um único sistema. Portanto, observa-se a importância da comunicação e do fluxo de informações. Visando a coordenação e a integração dos processos logísticos, Singh et al. (2019) sugerem a utilização de tecnologias como a internet das coisas e computação em nuvem como ferramentas de auxílio à gestão da cadeia de suprimentos.

Para Galindo (2016), é possível aplicar, aos processos logísticos e à gestão da cadeia de suprimentos, sensores inteligentes e softwares que coletam, processam e transmitem

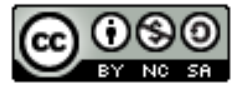


informações em tempo real por meio de sistemas cyber-físicos, atingindo um nível de automação onde os equipamentos e os humanos envolvidos possam se comunicar. Barreto et al. (2017) afirmam que a introdução desses conceitos pode impactar aspectos como o planejamento de recursos, a gestão de armazéns e a gestão e operação de sistemas de transportes.

De acordo com Tjahjono et al. (2017), apesar dos inúmeros benefícios que a Indústria 4.0 é capaz de proporcionar à logística e à gestão da cadeia de suprimentos, as novas tecnologias podem causar efeitos indesejados aos processos, caso sejam implementados sem os devidos estudos ou de maneira precipitada, evidenciando a necessidade de expansão de estudos sobre o tema.

No Brasil, existe a Agenda Brasileira para a Indústria 4.0, criada pelo Ministério da Indústria, Comércio Exterior e Serviços (MDIC), que tem como objetivo contribuir para a transformação das empresas em direção à Indústria 4.0, por meio de medidas que consideram o grau de maturidade ou necessidade de cada empresa. Apesar disso, de acordo com o relatório "Readiness for the Future of Production Report 2018" (World Economic Forum, 2018) o país segue atrasado quanto à sua estrutura de produção em relação aos países líderes de mercado, como Estados Unidos, Alemanha e Japão.

\section{Metodologia}

O procedimento metodológico deste artigo aplica uma abordagem de revisão bibliométrica e sistemática para mapeamento dos principais estudos sobre o assunto averiguado, coletados por meio de buscas diretas na base de dados Web of Science, que é a mais equilibrada em termos de abrangência, anos de cobertura e cobertura internacional (Chen, 2010), com abrangência nacional e internacional.

De acordo com Bastos et al. (2019), a bibliometria é um processo de contagem de atividades bibliográficas, que possibilita o estudo de variáveis específicas, como autores, instituições, quantidade de citações, dentre outras que possam vir a ser de interesse do pesquisador. De forma geral, a bibliometria caracteriza-se como "o estudo dos aspectos quantitativos da produção, disseminação, e uso da informação registrada, a partir de padrões e modelos matemáticos (Sciasci et al., 2012).

Já a análise sistemática, caracteriza-se, segundo Thorpe et al. (2005), por empregar uma metodologia de pesquisa com rigor científico e de grande transparência, cujo objetivo visa minimizar o enviesamento da literatura, na medida em que é feita uma recolha exaustiva dos textos publicados sobre o tema em questão.

A metodologia utilizada baseou-se em De Abreu e De Souza (2019) e consiste em três etapas: (i) planejamento da revisão; (ii) realização da revisão; e (iii) comunicação e divulgação. $\mathrm{Na}$ primeira etapa, inicialmente se identificou a necessidade da revisão e, em seguida, foi desenvolvido um protocolo de buscas.

Para a realização da pesquisa, optou-se pela utilização do termo $T S=(("$ industry 4.0 " AND "logistic*") OR ("industry 4.0" AND "supply chain*") OR ("logistic* 4.0")), que identifica as

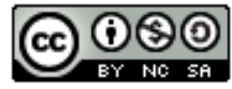


palavras encontradas nos títulos, palavras-chave e resumos dos artigos indexados na base de dados. Destaca-se que se optou por utilizar os termos em inglês para dar maior abrangência ao estudo. Isso porque mesmo pesquisas publicadas em outras línguas como português e espanhol apresentam, geralmente, resumo em inglês, ou seja, abstract. Quanto aos índices de citação, foram considerados SCI-EXPANDED, SSCI, A\&HCI, CPCI-S, CPCI-SSH e ESCI, sem restrições. A Tabela 1 demonstra de maneira resumida os critérios de pesquisa utilizados.

Tabela 1. Critérios de busca utilizados na pesquisa

\begin{tabular}{cc}
\hline Critério & Descrição \\
\hline Tópico & $T S=(($ "industry 4.0" AND "logistic*") OR ("industry 4.0" \\
AND "supply chain*”) OR ("logistic* 4.0”)) \\
Web of Science
\end{tabular}

Inicialmente, a busca direta na base de dados encontrou 420 artigos, porém os seguintes procedimentos foram realizados: (i) avaliação do título do artigo; (ii) avaliação do resumo e das palavras-chave do artigo; e (iii) leitura completa do artigo. Na etapa de leitura completa do artigo, foram avaliados critérios como a consistência da revisão bibliográfica apresentada, a aderência do estudo com os objetivos propostos e o tratamento dos resultados. Consequentemente, a quantidade de artigos incluídos no repositório de pesquisa reduziu para 136 publicações.

Por fim, na etapa de comunicação e divulgação, que consiste na apresentação de relatórios e apresentação de resultados, os seguintes aspectos foram considerados: (i) quantidade de publicações por ano; (ii) quantidade de citações por ano; (iii) principais publicações (aquelas mais citadas); (iv) autores de destaque; (v) publicações por país; e (vi) principais periódicos. Ademais, as publicações brasileiras foram avaliadas quanto aos seus objetivos, contribuições para o tema e aplicabilidade, estratégia ainda em falta na literatura.

\section{REVISÃO BIBLIOGRÁFICA}

A partir da base Web of Science, foram realizadas as análises bibliométricas (dos estudos incluídos no repositório de pesquisa) e sistemáticas (dos estudos brasileiros) expostas nas próximas seções.

\subsection{ANÁliSE BIBLIOMÉTRICA DAS PUBLICAÇõES SELECIONADAS}

Ao fim da análise preliminar, 136 publicações foram selecionadas para a pesquisa. A partir daí, foram realizadas análises bibliométricas dos artigos destacados e por fim, análises sistemáticas dos estudos brasileiros.

A Figura 1 mostra a evolução das publicações ao longo dos anos. Nela, é possível identificar o surgimento do tema no ano de 2015 e sua crescente evolução até o ano de 2020. Os

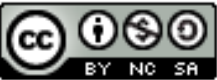



uma revisão da literatura. Brazilian Journal of Production Engineering, 7(2), 128-142.

primeiros registros de aplicações da Indústria 4.0 na logística e na cadeia de suprimentos se dão apenas em 2015, porém o rápido crescimento da curva indica o desenvolvimento e popularização do tema nos anos seguintes.

Figura 1. Quantidade de publicações por ano.

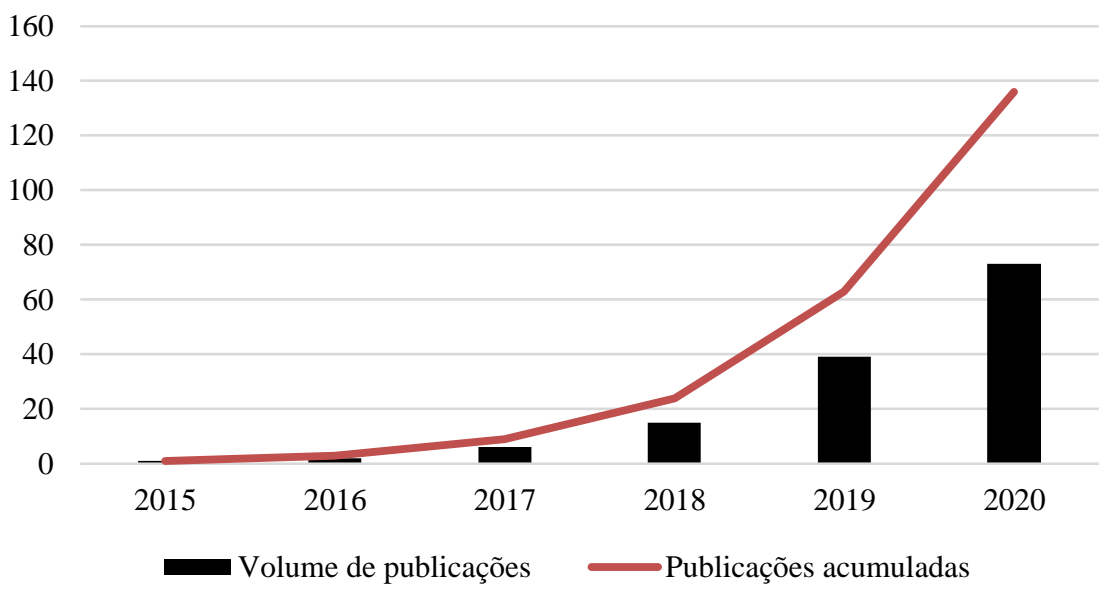

Posteriormente, foram analisadas também as quantidades de citações totais por ano. Os resultados da análise estão expressos na Figura 2. Nela, é possível identificar um crescimento no número de citações totais por ano, tal qual o de publicações expresso anteriormente, inclusive com pico de publicações em 2020.

Figura 2. Quantidade de citações por ano.

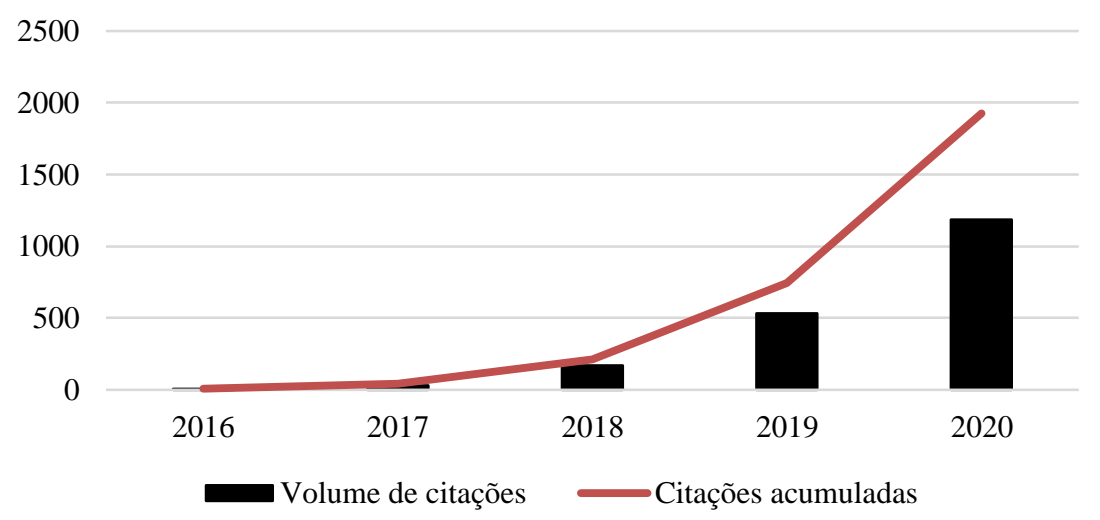

Além disso, cabe destacar também os estudos mais relevantes do repositório de pesquisa. Dentre os artigos selecionados, destacam-se os expressos na Tabela 2, que são os mais citados até o momento da realização desta pesquisa. As publicações mais citadas são: Hofmann e Ruesch (2017), com 338 citações; Ivanov et al. (2016), com 144 citações; e Ivanov et al. (2019), com 144 citações. Já em relação à média de citações por ano, os principais artigos são Hofmann e Ruesch (2017), com média igual a 85; Dolgui et al. (2020), com média igual a 75; e Ivanov et al. (2019), com a média de 72 citações, como pode ser observado na Tabela 2.

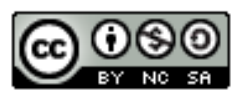


Citação (APA): Souza, G. M. de, Abreu, V. H. S. de, \& D'Agosto, M. de A. (2021). Indústria 4.0 aplicada à gestão da cadeia de suprimentos: uma revisão da literatura. Brazilian Journal of Production Engineering, 7(2), 128-142.

Tabela 2. Relação de publicações mais citadas.

\begin{tabular}{|c|c|c|c|}
\hline Título & Referência & Citações & $\begin{array}{l}\text { Média de } \\
\text { Citações por } \\
\text { Ano }\end{array}$ \\
\hline $\begin{array}{c}\text { Industry } 4.0 \text { and the current status as well as future } \\
\text { prospects on logistics }\end{array}$ & $\begin{array}{l}\text { Hofmann e Ruesch } \\
\qquad(2017)\end{array}$ & 338 & 85 \\
\hline $\begin{array}{l}\text { A dynamic model and an algorithm for short-term supply } \\
\text { chain scheduling in the smart factory industry } 4.0\end{array}$ & Ivanov et al. (2016) & 186 & 37 \\
\hline $\begin{array}{l}\text { The impact of digital technology and Industry } 4.0 \text { on the } \\
\text { ripple effect and supply chain risk analytics }\end{array}$ & Ivanov et al. (2019) & 144 & 72 \\
\hline $\begin{array}{c}\text { Scheduling in production, supply chain and Industry } 4.0 \\
\text { systems by optimal control: fundamentals, state-of-the-art } \\
\text { and applications }\end{array}$ & Dolgui et al. (2019) & 86 & 43 \\
\hline $\begin{array}{l}\text { Evaluating challenges to Industry } 4.0 \text { initiatives for } \\
\text { supply chain sustainability in emerging economies }\end{array}$ & $\begin{array}{l}\text { Luthra e Mangla } \\
\qquad(2018)\end{array}$ & 85 & 28 \\
\hline $\begin{array}{l}\text { Blockchain-oriented dynamic modelling of smart contract } \\
\text { design and execution in the supply chain }\end{array}$ & Dolgui et al. (2020) & 75 & 75 \\
\hline $\begin{array}{c}\text { New logistics and production trends as the effect of global } \\
\text { economy changes }\end{array}$ & $\begin{array}{l}\text { Kovacs e Kot } \\
\quad(2016)\end{array}$ & 57 & 11 \\
\hline $\begin{array}{l}\text { Towards industry } 4.0 \text { mapping digital technologies for } \\
\text { supply chain management-marketing integration }\end{array}$ & Ardito et al. (2019) & 51 & 26 \\
\hline $\begin{array}{l}\text { Design and application of Internet of things-based } \\
\text { warehouse management system for smart logistics }\end{array}$ & Lee et al. (2018) & 51 & 17 \\
\hline Logistics 4.0 and emerging sustainable business models & $\begin{array}{l}\text { Strandhagen } e t \\
\quad \text { al. }(2017)\end{array}$ & 36 & 9 \\
\hline
\end{tabular}

Além disso, dentre as publicações analisadas, é possível observar destaque de dois autores que são: Ivanov, D e Dolgui, A, que além de aparecerem duas vezes cada entre as publicações mais citadas, expostas na Tabela 2, são, também, os dois autores com mais publicações sobre o tema, como pode ser identificado na Tabela 3.

Tabela 3. Relação de autores com mais publicações.

\begin{tabular}{cc}
\hline Autor & Publicações \\
\hline Ivanov, D. & 6 \\
Dolgui, A. & 5 \\
Mangla, S. & 5 \\
Bag, S. & 4 \\
Luthra, S. & 4 \\
Sokolov, B. & 4 \\
Strandhagen, J. & 4 \\
Dhamija, P. & 3 \\
Dias, L. & 3 \\
Garza-Reyes, J. & 3 \\
\hline
\end{tabular}

Ainda em relação aos principais autores, observa-se a predominância de autores estrangeiros. Nenhum autor brasileiro está entre os principais pesquisadores do tema em relação às publicações selecionadas no estudo.

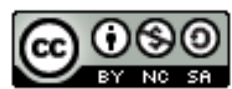


Entretanto, a análise da quantidade de publicações por país, exposta na Figura 3, mostra uma quantidade considerável de publicações nacionais, ocupando a quinta posição do ranking com 9\% das publicações. Além disso, pela Figura 3, nota-se que os países que mais publicam estudos sobre o assunto são Inglaterra, com $17 \%$ das publicações, e Alemanha e Índia, com $13 \%$ das publicações cada.

Figura 3. Quantidade de publicações por país.

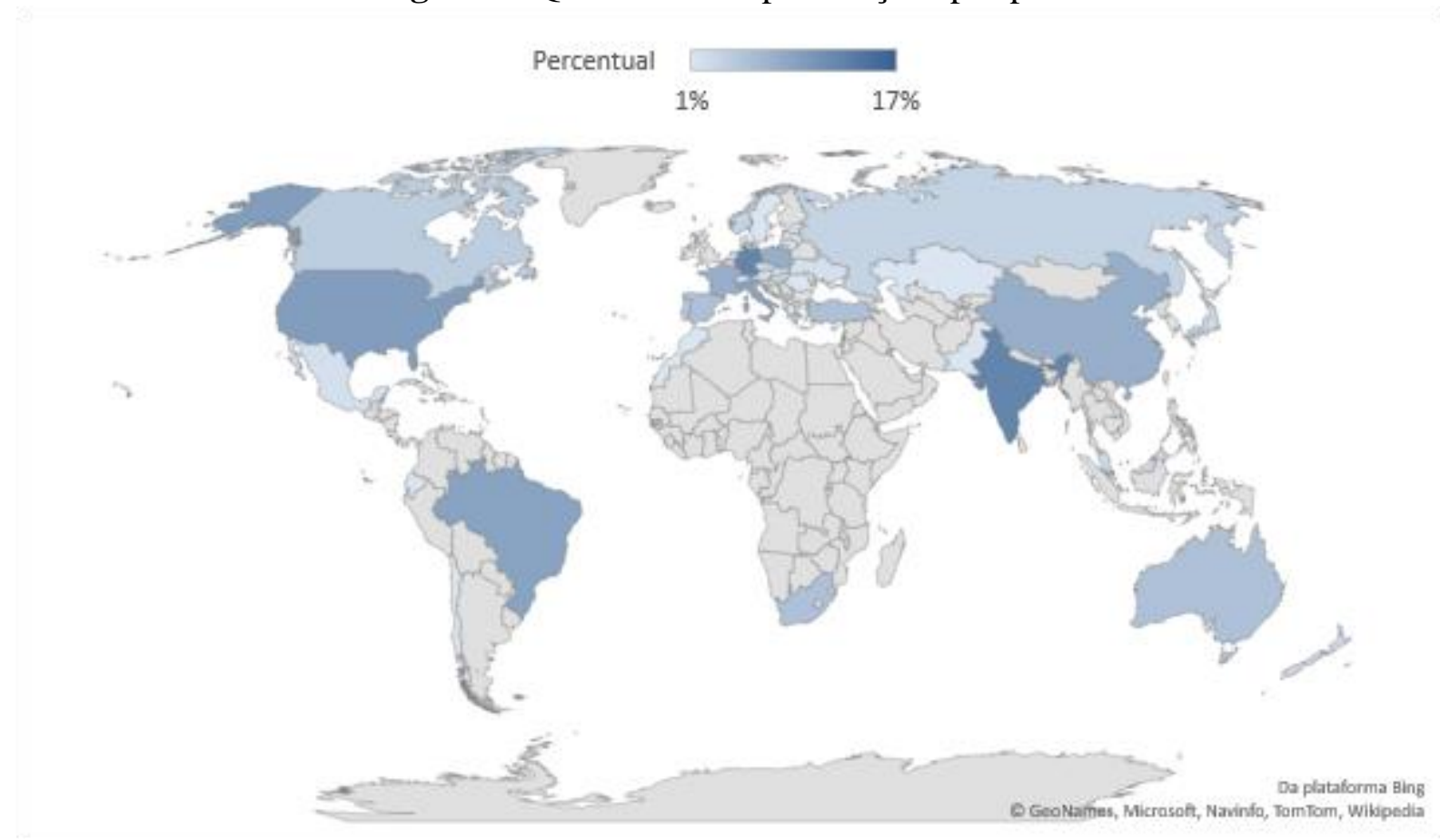

Cabe mencionar também que é de suma importância avaliar os artigos por periódico de publicação e o fator de impacto correspondentes, de modo a compreender quais revistas tem maior afinidade com o assunto e a relevância de cada uma delas. As principais revistas e seus respectivos fatores de impacto são exibidos na Tabela 4.

Tabela 4. Principais periódicos

\begin{tabular}{lcc}
\hline \multicolumn{1}{c}{ Periódico } & Publicações & Fator de Impacto \\
\hline International Journal of Production Research & 10 & 4.577 \\
Production Planning and Control & 9 & 3.605 \\
International Journal of Production Economics & 6 & 5.134 \\
Resources Conservation and Recycling & 5 & 8.086 \\
Sustainability & 5 & 2.576 \\
Computers in Industry & 4 & 3.954 \\
Journal of Cleaner Production & 3 & 7.246 \\
Computers Industrial Engineering & 3 & 4.135 \\
Journal of Manufacturing Technology Management & 3 & 3.385 \\
Industrial Management and Data Systems & 3 & 3.329 \\
\hline
\end{tabular}

Em relação à quantidade de publicações, observa-se na Tabela 4 que os principais periódicos são: o International Journal of Production Research, com 10 publicações sobre o tema; o

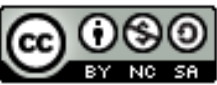


Citação (APA): Souza, G. M. de, Abreu, V. H. S. de, \& D'Agosto, M. de A. (2021). Indústria 4.0 aplicada à gestão da cadeia de suprimentos: uma revisão da literatura. Brazilian Journal of Production Engineering, 7(2), 128-142.

Production Planning and Control, com 9 publicações; e o International Journal of Production Economics, com 6. Tratando-se do fator de impacto, o principal periódico envolvido na observação é o Resources Conservation and Recycling, que apresenta fator 8,086, seguido do Journal of Cleaner Production, que tem fator 7,246, ambos com excelentes pontuações.

\subsection{ANÁLISE DAS PUBLICAÇÕES NACIONAIS}

Além das análises bibliométricas realizadas anteriormente, foram analisadas também as publicações onde houve participação de autores brasileiros. No total foram identificados 10 artigos, expostos na Tabela 5.

Tabela 5. Publicações nacionais presentes na pesquisa

\begin{tabular}{|c|c|c|c|}
\hline Título & Referência & Periódico & $\begin{array}{l}\text { Total de } \\
\text { Citações }\end{array}$ \\
\hline $\begin{array}{c}\text { Hybrid approach for the integrated } \\
\text { scheduling of production and transport } \\
\text { processes along supply chains }\end{array}$ & $\begin{array}{l}\text { Frazzon et al. } \\
\qquad(2018)\end{array}$ & $\begin{array}{l}\text { International Journal of } \\
\text { Production Research }\end{array}$ & 28 \\
\hline $\begin{array}{c}\text { The healthcare sustainable supply chain } \\
\text { 4.0: The circular economy transition } \\
\text { conceptual framework with the corporate } \\
\text { social responsibility mirror }\end{array}$ & $\begin{array}{l}\text { Dau et al. } \\
\text { (2019) }\end{array}$ & Sustainability & 15 \\
\hline $\begin{array}{c}\text { Supply chain } 4.0: \text { concepts, maturity and } \\
\text { research agenda }\end{array}$ & $\begin{array}{l}\text { Frederico et al. } \\
\qquad(2020)\end{array}$ & $\begin{array}{l}\text { Supply Chain Management - } \\
\text { An International Journal }\end{array}$ & 8 \\
\hline $\begin{array}{c}\text { Industry } 4.0 \text { and digital supply chain } \\
\text { capabilities: A framework for } \\
\text { understanding digitalisation challenges } \\
\text { and opportunities }\end{array}$ & $\begin{array}{l}\text { Queiroz et al. } \\
\qquad(2019)\end{array}$ & $\begin{array}{l}\text { Benchmarking - An } \\
\text { International Journal }\end{array}$ & 6 \\
\hline Towards supply chain management 4.0 & $\begin{array}{l}\text { Frazzon et al. } \\
\qquad(2019)\end{array}$ & $\begin{array}{c}\text { Brazilian Journal of } \\
\text { Operations \& Production } \\
\text { Management }\end{array}$ & 6 \\
\hline $\begin{array}{c}\text { The moderating effect of Industry } 4.0 \text { on } \\
\text { the relationship between lean supply chain } \\
\text { management and performance } \\
\text { improvement }\end{array}$ & $\begin{array}{l}\text { Tortorella et al. } \\
\qquad \text { (2019) }\end{array}$ & $\begin{array}{l}\text { Supply Chain Management - } \\
\text { An International Journal }\end{array}$ & 6 \\
\hline $\begin{array}{l}\text { How connectivity and search for } \\
\text { producers impact production in industry } \\
4.0 \text { networks }\end{array}$ & $\begin{array}{l}\text { Pereira et al. } \\
\quad(2018)\end{array}$ & $\begin{array}{c}\text { Brazilian Journal of } \\
\text { Operations \& Production } \\
\text { Management }\end{array}$ & 4 \\
\hline $\begin{array}{c}\text { Performance measurement for supply } \\
\text { chains in the Industry } 4.0 \text { era: a balanced } \\
\text { scorecard approach }\end{array}$ & $\begin{array}{l}\text { Frederico et al. } \\
\qquad(2020)\end{array}$ & $\begin{array}{c}\text { International Journal of } \\
\text { Productivity and Performance } \\
\text { Management }\end{array}$ & 2 \\
\hline $\begin{array}{c}\text { Method to assess the adherence of internal } \\
\text { logistics equipment to the concept of CPS } \\
\text { for industry } 4.0\end{array}$ & $\begin{array}{l}\text { Matana et al. } \\
\quad(2020)\end{array}$ & $\begin{array}{l}\text { International Journal of } \\
\text { Production Economics }\end{array}$ & 0 \\
\hline $\begin{array}{c}\text { Industry } 4.0 \text { and the supply chain } \\
\text { digitalisation: a blockchain diffusion } \\
\text { perspective }\end{array}$ & $\begin{array}{c}\text { Wamba e } \\
\text { Queiroz (2020) }\end{array}$ & $\begin{array}{l}\text { Production Planning \& } \\
\text { Control }\end{array}$ & 0 \\
\hline
\end{tabular}

Frazzon et al. (2018) e Dau et al. (2019) são as principais publicações nacionais, com 28 e 15 citações, respectivamente. Já Frederico et al. (2020), Matana et al. (2020) e Wamba e Queiroz (2020) não apresentavam nenhuma citação no momento de desenvolvimento dessa pesquisa, porém é importante destacar que são publicações recentes e futuramente podem estar em uma posição de maior relevância. 
Frederico et al. (2020), Queiroz et al. (2019) e Frazzon et al. (2019) apresentam um panorama da logística 4.0 e da digitalização da cadeia de suprimentos, destacando os principais conceitos e as principais tecnologias empregadas, além de apresentar possíveis benefícios e desafios futuros para o setor logístico. Frederico et al. (2020) ressaltam a relevância da internet das coisas, enquanto Frazzon et al. (2019) também citam a importância do big data e da computação em nuvem. Já Queiroz et al. (2019) afirmam que as tecnologias da informação e comunicação de maneira geral serão fundamentais para a logística, mas ressaltam que o fator humano ainda será o recurso mais importante e por isso, necessita do devido investimento e treinamento para se adaptar as mudanças futuras.

Frazzon et al. (2018) e Pereira et al. (2018) utilizam modelos de simulação para testar a aplicabilidade das novas tecnologias em processos produtivos e logísticos. Frazzon et al. (2018) simulam um modelo de planejamento integrado de produção e transportes, controlado em tempo real com auxílio de sistemas cyber-físicos. Os autores mostram que, apesar do experimento não ter sido realizado em um ambiente real, a tecnologia foi capaz de aumentar a capacidade de tomada de decisões em situações de contingência e de reduzir a quantidade de ordens em atraso. Também com o auxílio da simulação, Pereira et al. (2018) avaliam uma cadeia de suprimentos genérica através de uma rede de grafos e concluem que os sistemas cyber-físicos permitem maior integração entre as diferentes partes envolvidas no processo, pois no estudo, uma maior quantidade de nós foi alcançada em menor tempo após a utilização da tecnologia.

Tortorella et al. (2019) relacionam a Indústria 4.0 com a logística lean em empresas brasileiras. Os autores afirmam que apesar da possibilidade de se obter benefícios financeiros e operacionais nesse processo, a mudança de mentalidade dos agentes envolvidos pode ser necessária em alguns casos. Entretanto, os autores afirmam também que essa é uma característica regional e que diferentes países podem se adaptar de maneiras diferentes. Wamba e Queiroz (2020) também citam a regionalização em seu estudo sobre a aplicação do blockchain na cadeia de suprimentos, pois ao analisar a maturidade da digitalização logística de empresas dos Estados Unidos e da Índia, afirmam que países diferentes apresentam níveis diferentes de evolução tecnológica, além de apresentarem necessidades e desafios diferentes.

Dau et al. (2019) aplicam a internet das coisas nas operações de logística reversa de um hospital privado do Rio de Janeiro, buscando a economia circular. Os autores afirmam que a utilização dessa tecnologia pode trazer, além dos benefícios ambientais, benefícios sociais e econômicos para o hospital analisado, bem como para a sociedade como um todo. Entretanto, assim como Frazzon et al. (2019), indicam que apesar dos inúmeros benefícios possíveis, ainda é necessário desenvolver as tecnologias para que estas possam atingir todo o seu potencial.

Frederico et al. (2020) e Matana et al. (2020) avaliam as possíveis melhorias de desempenho logístico geradas pela utilização de tecnologias disruptivas. Entretanto, enquanto Frederico et al. (2020) estudam toda a cadeia de suprimentos, utilizando o método Balanced Scorecard (BSC), Matana et al. (2020) concentram os estudos, com base em redes neurais artificias, na aplicação de internet das coisas e de sistemas cyber-físicos em equipamentos de logística 
interna, como empilhadeiras e esteiras. Os autores concluem que as novas tecnologias são aplicáveis à logística e que podem contribuir também com outras áreas, como saúde, mobilidade urbana e produção. Frederico et al. (2020) ressaltam que as tecnologias estudadas devem ser integradas vertical e horizontalmente, visando a interoperabilidade que, segundo eles, é o principal aspecto da quarta revolução industrial.

\section{CONCLUSÕES}

Devido ao crescimento e popularização de pesquisas relacionadas à Indústria 4.0, foi constatada a necessidade de estudo da sua aplicação na logística e na cadeia de suprimentos. Assim, esse artigo teve como objetivo estudar as principais publicações referentes ao tema. Portanto, foram realizadas análises bibliométricas e sistemáticas dessas publicações, visando orientar pesquisadores sobre artigos relacionados ao tema proposto.

As análises bibliométricas, apesar da pequena amostra obtida, demonstram a expansão do tema desde o seu surgimento, em 2015, tendo seu ápice, até o momento, no ano de 2020. Demonstram também o crescimento no número de citações, que vem acompanhando a quantidade de publicações e também teve seu auge no ano de 2020.

É importante destacar também a relevância das pesquisas brasileiras no assunto, que aparecem em quinto lugar em número de publicações, com 10, ficando atrás apenas de Inglaterra, Alemanha, Índia e Estados Unidos. Em relação aos autores, destacam-se, principalmente Ivanov, D.; Dolgui, A.; e Mangla, S..

As análises sistemáticas das publicações brasileiras mostram uma tendência em analisar, dentre as tecnologias da Indústria 4.0, a utilização da internet das coisas e dos sistemas cyberfísicos como facilitadores da comunicação e integração entre envolvidos nos processos de distribuição e como ferramenta de controle de processos operacionais.

De acordo com as publicações brasileiras, a implementação de novas tecnologias nos processos de distribuição pode contribuir de forma significativa em questões econômicas, operacionais e até mesmo ambientais. Como benefícios, os autores citam o aumento no nível de serviço das operações logísticas, a redução de estoques, o uso sustentável de matérias primas e a otimização de rotas de entrega.

Outro aspecto fundamental é a diversificação de etapas da cadeia de suprimentos analisadas pelos autores, que vão desde planejamento de atividades estratégicas, como o relacionamento com fornecedores, até o controle e planejamento de rotas de transporte. Observa-se também que os exemplos de aplicação não se restringem a um setor específico da indústria, pois os artigos estudaram casos genéricos, que podem ser replicados em outros ramos.

Apesar de o estudo indicar avanços nas pesquisas e sugerir que a implementação de novas tecnologias é benéfica para os sistemas de distribuição, pode-se afirmar que, como citado anteriormente, a quantidade de estudos sobre o tema ainda é muito pequena e são necessários maiores investimentos na área para que novas descobertas sejam realizadas e possa se concluir efetivamente os impactos da Indústria 4.0 na logística.

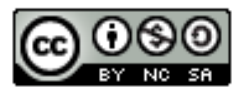


Citação (APA): Souza, G. M. de, Abreu, V. H. S. de, \& D'Agosto, M. de A. (2021). Indústria 4.0 aplicada à gestão da cadeia de suprimentos: uma revisão da literatura. Brazilian Journal of Production Engineering, 7(2), 128-142.

Recomenda-se que futuramente esse estudo seja replicado, tendo em vista o crescimento do tema, e que as análises sistemáticas sejam expandidas aos artigos internacionais, tendo como finalidade incorporar novas técnicas na realidade brasileira e analisar o desenvolvimento dos processos em outros ambientes.

É importante ressaltar também que a escolha das palavras-chave utilizadas na busca e o banco de dados escolhido tem influência direta no resultado da pesquisa. Para estudos futuros é recomendada a utilização de novos termos de busca e de outros repositórios científicos, visando a obtenção de novos resultados que a presente pesquisa não foi capaz de atingir, dadas as suas limitações.

\section{REFERENCIAS BIBLIOGRÁFICAS}

Ardito, L., Petruzzelli, A. M., Panniello, U., \& Garavelli, A. C. (2019). Towards Industry 4.0: Mapping digital technologies for supply chain management-marketing integration. Business Process Management Journal, 25(2), 323-346. https://doi.org/10.1108/BPMJ-04-2017-0088

Arnold, C., Kiel, D., \& Voigt, K. I. (2017). Innovative business models for the industrial internet of things. BHM Berg-und Hüttenmännische Monatshefte, 162(9), 371-381. https://doi.org/10.1007/s00501-017-0667-7

Barreto, L., Amaral, A., \& Pereira, T. (2017). Industry 4.0 implications in logistics: an overview. Procedia Manufacturing, 13,

1245-1252.

https://doi.org/10.1016/j.promfg.2017.09.045

Bastos, M. H. R., de Oliveira, U. R., de Rezende Souza, T. C., dos Santos, R. F., \& do Lago, M. M. (2019). Análise de discurso e Análise de Conteúdo: Um levantamento de suas aplicações nas ciências aplicadas membros da Administração. Brazilian Journal of Development, 5(11), 26302-26322. https://doi.org/10.34117/bjdv5n11-265

Bauer, W., Hämmerle, M., Schlund, S., \& Vocke, C. (2015). Transforming to a hyperconnected society and economy-towards an "Industry 4.0". Procedia Manufacturing, 3, 417424. https://doi.org/10.1016/j.promfg.2015.07.200

BRASIL, Ministério da Indústria, Comércio Exterior e Serviços. Agenda Brasileira para a Indústria 4.0. Recuperado de http://www.industria40.gov.br/

Chen, W., Liu, W., Geng, Y., Brown, M. T., Gao, C., \& Wu, R. (2017). Recent progress on emergy research: A bibliometric analysis. Renewable and Sustainable Energy Reviews, 73, 1051-1060. https://doi.org/10.1016/j.rser.2017.02.041

Daú, G., Scavarda, A., Scavarda, L. F., \& Portugal, V. J. T. (2019). The healthcare sustainable supply chain 4.0: The circular economy transition conceptual framework with the corporate social responsibility mirror. Sustainability, 11(12), 3259. https://doi.org/10.3390/su11123259

de Abreu, V. H. S., \& de Souza, G. M. (2019). Uma revisão bibliométrica sobre a impressão 3D em concreto. Brazilian Journal of Production Engineering - BJPE, 5(5), 50-62.

Dolgui, A., Ivanov, D., Sethi, S. P., \& Sokolov, B. (2019). Scheduling in production, supply chain and Industry 4.0 systems by optimal control: fundamentals, state-of-the-art and applications. International Journal of Production Research,57(2), 411-432. https://doi.org/10.1080/00207543.2018.1442948

Dolgui, A., Ivanov, D., Potryasaev, S., Sokolov, B., Ivanova, M., \& Werner, F. (2020). Blockchain-oriented dynamic modelling of smart contract design and execution in the supply

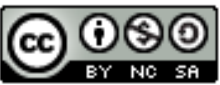

Esta obra está licenciada com uma Licença Creative Commons Atribuição-Não Comercial-Compartilha Igual 4.0 Internacional. Brazilian Journal of Production Engineering, São Mateus, Editora UFES/CEUNES/DETEC. 
Citação (APA): Souza, G. M. de, Abreu, V. H. S. de, \& D'Agosto, M. de A. (2021). Indústria 4.0 aplicada à gestão da cadeia de suprimentos: uma revisão da literatura. Brazilian Journal of Production Engineering, 7(2), 128-142.

chain. International Journal of Production Research,58(7), 2184-2199. https://doi.org/10.1080/00207543.2019.1627439

Engblom, J., Solakivi, T., Töyli, J., \& Ojala, L. (2012). Multiple-method analysis of logistics costs. International Journal of Production Economics, 137(1), 29-35. https://doi.org/10.1016/j.ijpe.2012.01.007

Erol, S., Jäger, A., Hold, P., Ott, K., \& Sihn, W. (2016). Tangible Industry 4.0: a scenariobased approach to learning for the future of production. Procedia CiRp, 54, 13-18. https://doi.org/10.1016/j.procir.2016.03.162

Fernandez, J. T., Jiménez, J. B. (2017). Supply chain integration and performance relationship: a moderating effects review. The International Journal of Logistics Management, 28(4), 1243-1271. https://doi.org/10.1108/IJLM-02-2016-0043

Frazzon, E. M., Albrecht, A., Pires, M., Israel, E., Kück, M., \& Freitag, M. (2018). Hybrid approach for the integrated scheduling of production and transport processes along supply chains. International Journal of Production Research,56(5), 2019-2035. https://doi.org/10.1080/00207543.2017.1355118

Frazzon, E. M., Rodriguez, C. M. T., Pereira, M. M., Pires, M. C., \& Uhlmann, I. (2019). Towards supply chain management 4.0. Brazilian Journal of Operations \& Production Management, 16(2), 180-191. https://doi.org/10.14488/BJOPM.2019.v16.n2.a2

Frederico, G. F., Garza-Reyes, J. A., Anosike, A., \& Kumar, V. (2019). Supply Chain 4.0: concepts, maturity and research agenda. Supply Chain Management: An International Journal, 25(2), 262-282. https://doi.org/10.1108/scm-09-2018-0339

Frederico, G. F., Garza-Reyes, J. A., Kumar, A., \& Kumar, V. (2020). Performance measurement for supply chains in the Industry 4.0 era: a balanced scorecard approach. International Journal of Productivity and Performance Management, 70(4), 789807. https://doi.org/10.1108/IJPPM-08-2019-0400

Galindo, L. D. (2016). The challenges of logistics 4.0 for the supply chain management and the information technology. Dissertação de mestrado, NTNU.

Hofmann, E., \& Rüsch, M. (2017). Industry 4.0 and the current status as well as future prospects on $\quad$ logistics. Computers industry, 89, 34. https://doi.org/10.1016/j.compind.2017.04.002

Ivanov, D., Dolgui, A., Sokolov, B., Werner, F., \& Ivanova, M. (2016). A dynamic model and an algorithm for short-term supply chain scheduling in the smart factory industry 4.0. International Journal of Production Research, 54(2), 386-402. https://doi.org/10.1080/00207543.2014.999958

Ivanov, D., Dolgui, A., \& Sokolov, B. (2019). The impact of digital technology and Industry 4.0 on the ripple effect and supply chain risk analytics. International Journal of Production Research, 57(3), 829-846. https://doi.org/10.1080/00207543.2018.1488086

Kagermann, H., Helbig, J., Hellinger, A., \& Wahlster, W. (2013). Recommendations for implementing the strategic initiative INDUSTRIE 4.0: Securing the future of German manufacturing industry; final report of the Industrie 4.0 Working Group. Forschungsunion.

Kang, H. S., Lee, J. Y., Choi, S., Kim, H., Park, J. H., Son, J. Y., Kim, B. H., \& Do Noh, S. (2016). Smart manufacturing: Past research, present findings, and future

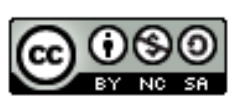


Citação (APA): Souza, G. M. de, Abreu, V. H. S. de, \& D'Agosto, M. de A. (2021). Indústria 4.0 aplicada à gestão da cadeia de suprimentos: uma revisão da literatura. Brazilian Journal of Production Engineering, 7(2), 128-142.

directions. International journal of precision engineering and manufacturing-green technology, 3(1), 111-128. https://doi.org/10.1007/s40684-016-0015-5

Kovács, G., \& Kot, S. (2016). New logistics and production trends as the effect of global economy changes. Polish Journal of Management Studies, 14. https://doi.org/10.17512/pjms.2016.14.2.11

Lasi, H., Fettke, P., Kemper, H. G., Feld, T., \& Hoffmann, M. (2014). Industry 4.0. Business \& information systems engineering, 6(4), 239-242. Recuperado de https://aisel.aisnet.org/bise/vol6/iss4/5

Lee, C. K. M., Lv, Y., Ng, K. K. H., Ho, W., \& Choy, K. L. (2018). Design and application of Internet of things-based warehouse management system for smart logistics. International Journal of Production Research, 56(8), 2753-2768. https://doi.org/10.1080/00207543.2017.1394592

Luthra, S., \& Mangla, S. K. (2018). Evaluating challenges to Industry 4.0 initiatives for supply chain sustainability in emerging economies. Process Safety and Environmental Protection, 117, 168-179. https://doi.org/10.1016/j.psep.2018.04.018

Matana, G., Simon, A., Godinho Filho, M., \& Helleno, A. (2020). Method to assess the adherence of internal logistics equipment to the concept of CPS for industry 4.0. International Journal of Production Economics, 228, 107845. https://doi.org/10.1016/j.ijpe.2020.107845

Melnyk, S. A., Lummus, R. R., Vokurka, R. J., Burns, L. J., \& Sandor, J. (2009). Mapping the future of supply chain management: a Delphi study. International journal of production Research, 47(16), 4629-4653. https://doi.org/10.1080/00207540802014700

Pedroso, M. C., \& Nakano, D. (2009). Knowledge and information flows in supply chains: A study on pharmaceutical companies. International journal of production economics, 122(1), 376-384. https://doi.org/10.1016/j.ijpe.2009.06.012

Pereira, A., de Oliveira Simonetto, E., Putnik, G., \& de Castro, H. C. G. A. (2018). How connectivity and search for producers impact production in Industry 4.0 networks. Brazilian Journal of Operations \& Production Management, 15(4), 528-534. https://doi.org/10.14488/BJOPM.2018.v15.n4.a6

Prajogo, D., \& Sohal, A. (2013). Supply chain professionals: A study of competencies, use of technologies, and future challenges. International Journal of Operations \& Production Management, 33(11-12), 1532-1554. https://doi.org/10.1108/IJOPM-08-2010-0228

Premm, M., \& Kirn, S. (2015). A multiagent systems perspective on Industry 4.0 supply networks. In German Conference on Multiagent System Technologies, Springer, Cham.

Queiroz, M. M., Pereira, S. C. F., Telles, R., \& Machado, M. C. (2019). Industry 4.0 and digital supply chain capabilities: A framework for understanding digitalisation challenges and opportunities. Benchmarking: An International Journal, 28(5), 1761-1782. https://doi.org/10.1108/BIJ-12-2018-0435

Sciasci, V., Garcia, S. F. A., \& Galli, L. C. D. L. A. (2012). Posicionamento de marcas globais: um estudo bibliométrico da produção científica na área. Revista Brasileira de Marketing, 11(2), 67-93. https://doi.org/10.5585/remark.v11i2.2332

Singh, S., Ghosh, S., Jayaram, J., \& Tiwari, M. K. (2019). Enhancing supply chain resilience using ontology-based decision support system. International Journal of Computer Integrated Manufacturing, 32(7), 642-657. https://doi.org/10.1080/0951192x.2019.1599443 
Citação (APA): Souza, G. M. de, Abreu, V. H. S. de, \& D'Agosto, M. de A. (2021). Indústria 4.0 aplicada à gestão da cadeia de suprimentos: uma revisão da literatura. Brazilian Journal of Production Engineering, 7(2), 128-142.

Stock, T., \& Seliger, G. (2016). Opportunities of sustainable manufacturing in industry 4.0. Procedia Cirp, 40, 536-541. https://doi.org/10.1016/j.procir.2016.01.129

Strandhagen, J. O., Vallandingham, L. R., Fragapane, G., Strandhagen, J. W., Stangeland, A. B. H., \& Sharma, N. (2017). Logistics 4.0 and emerging sustainable business models. Advances in Manufacturing, 5(4), 359-369. https://doi.org/10.1007/s40436-017$\underline{0198-1}$

Strange, R., \& Zucchella, A. (2017). Industry 4.0, global value chains and international business. Multinational Business Review, 25(3), 174-184. https://doi.org/10.1108/mbr-05$\underline{2017-0028}$

Sung, T. K. (2018). Industry 4.0: a Korea perspective. Technological forecasting and social change, 132, 40-45. https://doi.org/10.1016/j.techfore.2017.11.005

Szozda, N. (2017). Industry 4.0 and its impact on the functioning of supply chains. Logforum, 13. https://doi.org/10.17270/J.LOG.2017.4.2

Thoben, K. D., Wiesner, S., \& Wuest, T. (2017). "Industrie 4.0" and smart manufacturing-a review of research issues and application examples. International journal of automation technology, 11(1), 4-16. https://doi.org/10.20965/ijat.2017.p0004

Thorpe, R., Holt, R., Macpherson, A., \& Pittaway, L. (2005). Using knowledge within small and medium-sized firms: A systematic review of the evidence. International Journal of Management Reviews, 7(4), 257-281. https://doi.org/10.1111/j.1468-2370.2005.00116.x

Tjahjono, B., Esplugues, C., Ares, E., \& Pelaez, G. (2017). What does industry 4.0 mean to supply chain? Procedia manufacturing, 13,

1175-1182. https://doi.org/10.1016/j.promfg.2017.09.191

Tortorella, G., Miorando, R., \& Mac Cawley, A. F. (2019). The moderating effect of Industry 4.0 on the relationship between lean supply chain management and performance improvement. Supply Chain Management-An International Journal,24(2), 301-314. https://doi.org/10.1108/SCM-01-2018-0041

Wamba, S. F., \& Queiroz, M. M. (2020). Industry 4.0 and the supply chain digitalisation: a blockchain diffusion perspective. Production Planning \& Control, 1-18. https://doi.org/10.1080/09537287.2020.1810756

World Economic Forum - WEF. Readiness for the Future of Production, Report 2018. Recuperado de http://www3.weforum.org/docs/FOP_Readiness_Report_2018.pdf 